\title{
Glomerular Deposition of Properdin in Acute and Chronic
}

\section{Glomerulonephritis with Hypocomplementemia}

\author{
N. Gunnar Westberg, George B. Naff, John T. Boyer, and \\ AlFred F. Michael \\ From the Department of Pediatrics, University of Minnesota, Minneapolis, \\ Minnesota 55455 and Department of Medicine, Case Western Reserve \\ University, Cleveland, Ohio 44106
}

\begin{abstract}
A B S T R A C T Kidney tissue from 97 patients was studied by immunofluorescent techniques using antiserum to purified properdin. All patients with acute poststreptococcal glomerulonephritis showed deposition of properdin and the third component of complement (C3), either as "humps" on the basement membrane, or in the mesangium. In all cases of chronic membranoproliferative glomerulonephritis, properdin and C3 were localized in the glomeruli, most commonly in a lobular pattern on the basement membrane. Activation of $\mathrm{C} 3$ by the properdin system may explain the depressed serum levels of C3 and terminal complement components even though levels of earlier components are normal, and the deposition of C3, often without immunoglobulins, in the kidneys of patients with acute glomerulonephritis or chronic membranoproliferative glomerulonephritis.
\end{abstract}

\section{INTRODUCTION}

The properdin system, described by Pillemer et al. in 1954 (1) and Pillemer in 1955 (2) has been shown to inactivate $\mathrm{C} 3$, after interaction with zymosan, without significant inactivation of the earlier complement components. This discovery lead to extensive experimental work, but the nature and biological role of properdin remained controversial. Properdin has not been shown to be important in the pathogenesis of human disease. However, in a recent publication by Gewurz et al (3), it was shown that serum levels of properdin were regularly decreased in patients with acute glomerulonephritis (AGN), ${ }^{1}$ and in approximately one-half of pa-

Dr. Boyer's present address is the Department of Internal Medicine, College of Medicine, Tucson, Arizona 85721.

Received for publication 25 August and in revised form 22 October 1970.

${ }^{1}$ Abbreviations used in this paper: AGN, acute glomerulonephritis; HCGN, hypocomplementemic glomerulonephritis; FITC, fluorescein isothiocyanate; SLE, systemic lupus erythematosus. tients and chronic membranoproliferative glomerulonephritis with hypocomplementemia (HCGN).

The present study was undertaken in order to clarify the possible involvement of properdin in immunologic kidney diseases, particularly those with decreased levels of serum complement. This investigation was made possible by the recent report of Pensky et al (4) which describes a method for obtaining highly purified human properdin.

\section{METHODS}

The diagnosis of specific renal diseases was made on the basis of clinical history, physical findings, laboratory analysis, and renal histopathology as outlined in previous publications $(5,6)$. Tissue obtained by kidney biopsy and nephrectomy over a 4 month period of time was studied by immunofluorescent and light microscopy. In addition, material obtained by percutaneous kidney biopsy from patients with AGN and HCGN during the previous 12 months and kept in isopentane at $-70^{\circ} \mathrm{C}$ was also studied. The tissue was used only if the immunofluorescent staining for IgG, $\beta_{1} C$, and fibrin had not changed during the period of storage compared to the staining of the fresh tissue. Tissue from 97 patients was available for this study. Rabbit antisera produced according to methods previously described $(7,8)$ were tagged with fluorescein isothiocyanate (FITC) by the method of Wood, Thompson, and Goldstein (9), as modified in our laboratory (8). Two FITC-tagged antisera to human $\mathrm{C} 3$ were used, one prepared by immunizing rabbits with human $\beta_{1} \mathrm{C}$ isolated according to Müller-Eberhard, Nilsson, and Aronsson (10) (referred to in this publication as anti- $\beta_{1} C$ ), the other with $\beta_{1} C$ absorbed to zymosan (11) (referred to as anti- $\beta_{1} \mathrm{C}-\mathrm{Z}$ ). Rabbit antisera to $\mathrm{Clq}$ (kindly provided by Dr. R. M. Stroud) and C4 (12) (kindly provided by Dr. H. Müller-Eberhard), and goat antisera to antigenic components of C3 (components $\mathrm{B}, \mathrm{A}$, and D) (13) (kindly provided by Dr. Clark West) were used for indirect staining with FITC-tagged goat anti-rabbit or rabbit anti-goat IgG in the in the second layer. Antiserum to properdin was prepared by injecting rabbits at multiple sites with $0.5 \mathrm{mg}$ human properdin, purified according to Pensky et al (4), mixed with complete Freund's adjuvant. After 5 wk a booster injection was given and the rabbits were bled on several occasions. IgG was isolated from the 
antiserum and labeled with $\operatorname{FITC}(8,9)$. The specificity of the anti-properdin, anti- $\beta_{1} C$ and anti- $\beta_{1} C-Z$ sera was studied by absorption, double diffusion in agar (14), and immunoelectrophoresis (15). For absorption, small volumes of the tagged IgG or untagged serum were mixed and incubated overnight at $4^{\circ} \mathrm{C}$ with the appropriate antigen in increasing amounts until the immunofluorescent staining or reaction in agar double diffusion became negative, or until the serum became too dilute for study. Clq (11S) was prepared as described by Hinz and Mollner (16).

$\beta_{1} \mathrm{C}$ globulin (C3) was a gift from Dr. Müller-Eberhard (17) and C5 from Dr. Ulf Nilsson (17). All tissues were stained with antisera to IgG, fibrin, $\beta_{1} \mathrm{C}$, and properdin. Kidney sections from patients with AGN and HCGN were also stained for IgM and complement factors $\mathrm{Clq}$ and $\mathrm{C} 4$. The stained sections were read as negative, trace, or $1+-3+$ "trace" indicating a staining just stronger than that of the background or of normal control tissue.

\section{RESULTS}

Specificity of antisera. After absorption of the FITClabeled antiserum to properdin with $15 \mu \mathrm{g}$ properdin per mg IgG, the antiserum no longer stained kidney tissue that reacted strongly with unabsorbed antiserum (Table I). The staining was not affected by absorption with $\beta_{1} \mathrm{C}, \mathrm{Clq}$, or C5. FITC-tagged antisera to $\beta_{1} \mathrm{C}$ (anti $-\beta_{1} C$ ) or to $\beta_{1} C$ prepared by absorption on zymosan (anti- $\beta_{1} \mathrm{C}-Z$ ) and diluted untagged antisera to $\mathrm{C} 1 \mathrm{q}, \mathrm{C} 4$, and antigenic determinants of $\mathrm{C} 3,(\mathrm{~A}, \mathrm{~B}$, and $\mathrm{D})$ did not show a decrease in their staining ability after absorption with high concentrations of properdin. Anti$\beta_{1} \mathrm{C}$, anti-C1q, anti-A, anti-B, and anti-D could all be absorbed out by their appropriate antigens. The staining by the anti- $\beta_{1} C-Z$ antiserum could not be abolished by absorption with $\beta_{1} \mathrm{C}$ or properdin, used alone or together. The results are summarized in Table I.
In double diffusion in agar, the antiserum to properdin gave one line with normal human serum showing a reaction of identity with the line formed between properdin and antiproperdin (Fig. $1 a$ ). Anti-properdin did not react with purified C1q (11S), C3 $\left(\beta_{1} C\right)$, or C5. The anti- $\beta_{1} C$ serum did not react with the purified properdin but the anti- $\beta_{1} C-Z$ gave a weak precipitation reaction with properdin (Fig. $1 b$ ) which could be removed by absorption with properdin (Fig. 1c). In immunoelectrophoresis against fresh or aged human serum, anti- $\beta_{1} C$ was found to react with $\beta_{1} C$ and $\beta_{1} A$, while anti- $\beta_{1} \mathrm{C}-Z$ reacted with $\beta_{1} \mathrm{~A}$ only.

In the staining of kidney tissue, it was repeatedly observed that many sections that stained strongly for properdin did not stain with one or more of the antisera to $\mathrm{IgG}, \mathrm{IgM}$, fibrin, $\mathrm{Clq}$, and $\mathrm{C} 4$ and vice versa. All properdin-positive glomeruli reacted with anti- $\beta_{1} C$ serum; however, renal tissue from patients with other diseases frequently contained $\beta_{1} \mathrm{C}$ but not properdin. Tubular cells and casts in the tubular lumina often stained for $\beta_{1} C$, but only in a few cases for properdin.

Results of immunofluorescent studies (Table II). All eight patients with chronic membranoproliferative glomerulonephritis with low serum levels of C3 showed positive glomerular staining for properdin. The staining had a characteristic lobular distribution in six cases (Fig. 2), and showed a coarse deposition of properdin on the basement membrane without clear lobulation in two other cases (Fig. 3); the staining for properdin had always the same distribution as that for $\beta_{1} \mathrm{C}$. Clq and $C 4$ were found in $3 / 5$ and $4 / 5$ biopsies respectively (Table III). In four cases, the staining for IgG was

TABLE I

Inhibition of Glomerular Fluorescence by Absorptions of Antisera with Various Antigens

\begin{tabular}{|c|c|c|c|c|c|}
\hline \multirow[b]{2}{*}{ Antisera used for immunofluorescence } & \multicolumn{5}{|c|}{ Antigens used for absorption } \\
\hline & $\begin{array}{l}\text { Proper- } \\
\text { din }\end{array}$ & $\mathrm{C} 3\left(\beta_{1} \mathrm{C}\right)$ & $\mathrm{Clq}$ & $\mathrm{C} 5$ & $\begin{array}{l}\text { Aged } \\
\text { serum }\end{array}$ \\
\hline Anti-properdin (FITC-tagged IgG) & 15 & $>900$ & $>600$ & $>1000$ & \\
\hline Anti- $\beta_{1} C$ (FITC-tagged IgG) & $>320$ & 75 & & & \\
\hline Anti- $\beta_{1} C-Z$ (FITC-tagged IgG) & $>230$ & $>700$ & & & \\
\hline Anti-C1q serum, diluted $1: 20$ & $>160$ & $\therefore$ & 40 & & \\
\hline Anti-C4 serum, diluted 1:20 & $>160$ & & & & \\
\hline Anti-A serum, diluted $1: 10$ & $>160$ & 40 & & & \\
\hline Anti-B serum, diluted $1: 10$ & $>160$ & $\because 40$ & & & \\
\hline Anti-D serum, diluted $1: 4$ & $>160$ & & & & 3 parts* \\
\hline \multicolumn{6}{|c|}{$\begin{array}{l}\text { The numbers indicate the amount of antigen necessary to completely remove the staining ability } \\
\text { of the tagged antiserum for glomeruli from patients with } \mathrm{HCGH} \text {, expressed as micrograms of } \\
\text { antigen per milligrams IgC or per milliliters of diluted untagged antiserum. The symbol }(>) \\
\text { indicates that the highest amount used did not diminish the staining. } \\
\text { * After addition of undiluted antiserum to three parts of human serum, the staining became } \\
\text { negative. }\end{array}$} \\
\hline
\end{tabular}




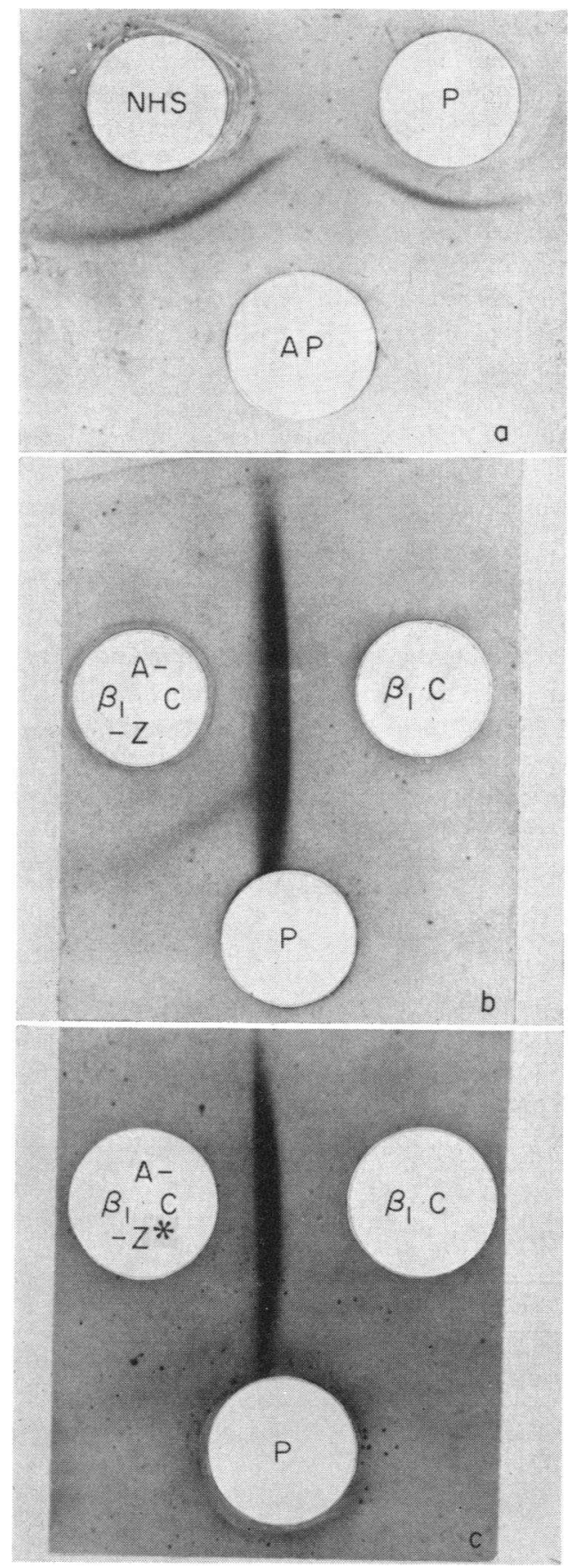

Figure 1 Double diffusion in agarose showing reactions of the anti-properdin and anti- $\beta_{1} \mathrm{C}-Z$ sera. (a) One line of identity is formed between anti-properdin serum $(A P)$, normal human serum $(N H S)$ and purified properdin $(P)$. (b) Antiserum to $\beta_{1} \mathrm{C}$ prepared with absorption of $\mathrm{C} 3$ to zymosan $\left(\mathrm{A}-\beta_{1} \mathrm{C}-\mathrm{Z}\right)$ gives a line when reacted with purified $\beta_{1} \mathrm{C}$ and a weak line with purified properdin $(P)$. (c) When anti- $\beta_{1} C-Z$ is absorbed with purified properdin $\left(A-\beta_{1} C-Z^{*}\right)$, the reaction with properdin completely disappears. completely negative and in three of these, the staining for IgM was also negative.

1 patient, a 27 year old man with an $11 \mathrm{yr}$ history of hematuria and decreasing kidney function, had the characteristic renal pathology of chronic membranoproliferative glomerulonephritis but had a normal serum complement. Immunofluorescent microscopy revealed the presence of $\beta_{1} C$ and properdin in the mesangium of the glomeruli, but no IgG was found. We have observed that the level of serum complement in several children with HCGN may increase to normal as they approach adulthood (5).

All eight patients with acute poststreptococcal glomerulonephritis had strongly positive glomerular fluorescence for properdin. In three cases the distribution was mostly peripheral with characteristic "humps" (Fig. 4) on the basement membrane while in the other five both $\beta_{1} \mathrm{C}$ and properdin were found predominantly in the mesangium (Fig. 5). This type of immunofluorescent pattern has been described for $\beta_{1} \mathrm{C}$ late in acute poststreptococcal glomerulonephritis (18). Three biopsies were negative for IgG and in no case was IgM found. Of patients with $\mathrm{AGN}$, one patient out of seven showed positive staining of the glomeruli for $\mathrm{C} 4$, but none for C1q. (Table III)

Tissue obtained on two occasions by kidney biopsy from one patient with subacute glomerulonephritis showed $1+$ mesangial deposition of properdin and $\beta_{1} \mathrm{C}$. This patient had evidence of a preceding streptococcal infection with elevated serum titers of antistreptococcal antibodies and the serum level of total hemolytic complement was low. He may very well represent a serious form of acute poststreptococcal nephritis.

1 of 11 patients with systemic lupus erythematosus (SLE) with nephritis showed $1+$ staining for properdin in deposits on the basement membrane and in some areas of the mesangium, while $\beta_{1} C$ was found with a $3+$ intensity only in the mesangium. 19 kidneys from patients with end stage renal failure, most of them removed before transplantation, were also stained for properdin. In two cases properdin was found together with C3 with an irregular focal deposition on the periphery of the capillary loops of a few glomeruli. One of those two patients had bilateral hydronephrosis as the cause of uremia, while the other patient was diagnosed clinically as having chronic glomerulonephritis. A pathological diagnosis was not possible on this end stage kidney.

In tissue from four patients with diabetes mellitus, linear staining of trace-positive intensity for properdin, and a $1-2+$ staining for $\beta_{1} C$ was seen on the basement membrane together with variable amounts of $\mathrm{IgG}$ and albumin. The kidney biopsy tissue from one patient with malignant hypertension showed a $2+$ deposition 

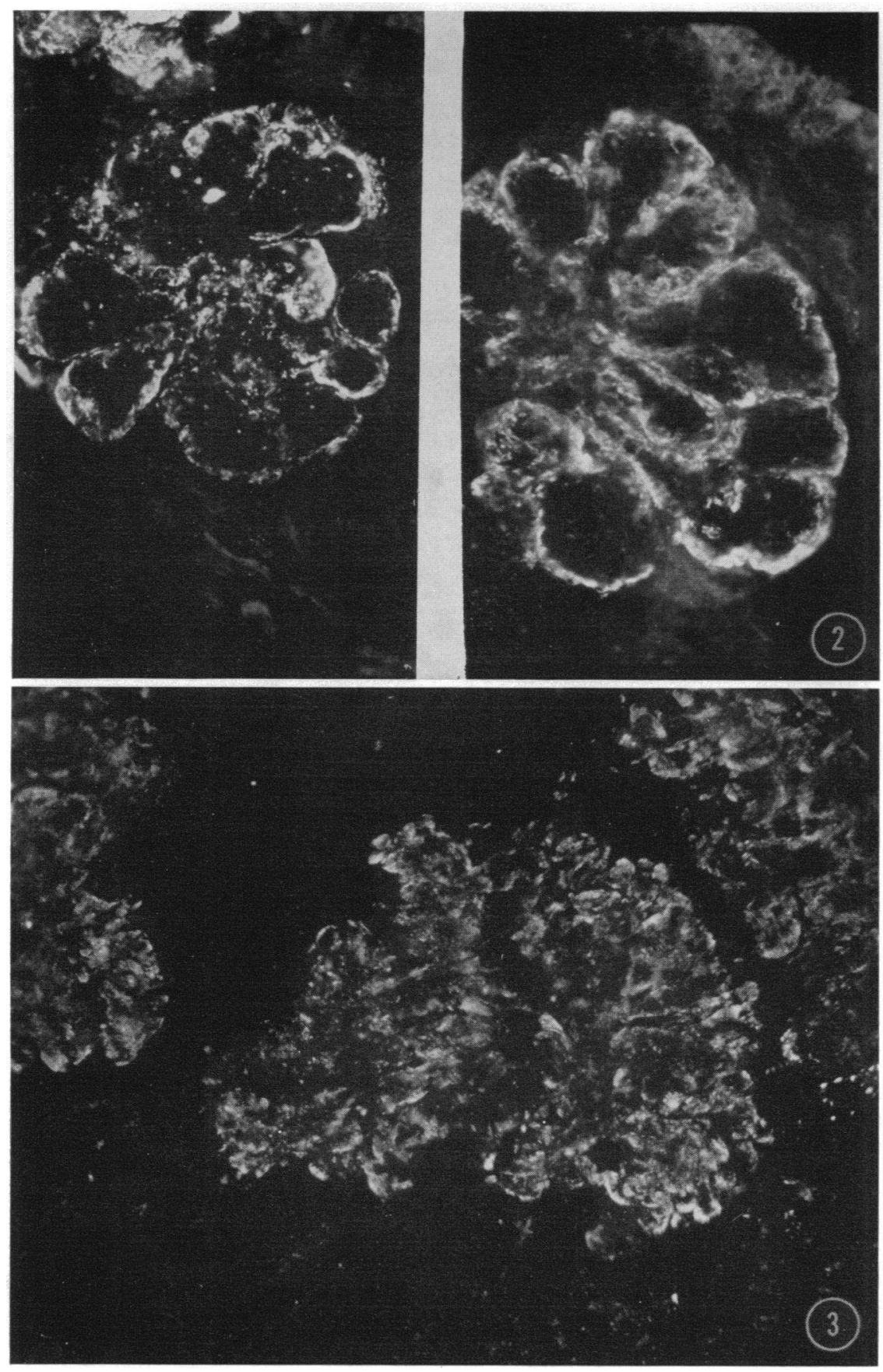

FIgURE 2 Section of kidney obtained from a patient with chronic membranoproliferative glomerulonephritis. The glomeruli show lobular staining for both properdin (left) and for $\beta_{1} \mathrm{C}$ (right). This pattern was seen in tissue from six out of eight patients. Fluorescent microscopy. $\times 400$.

Figure 3 Tissue from two patients with HCGH demonstrated coarse deposition of properdin (shown) and $\beta_{1} C$ on the glomerular basement membrane, without the lobular pattern noted in Fig. 2. Fluorescent microscopy. $\times 600$. 

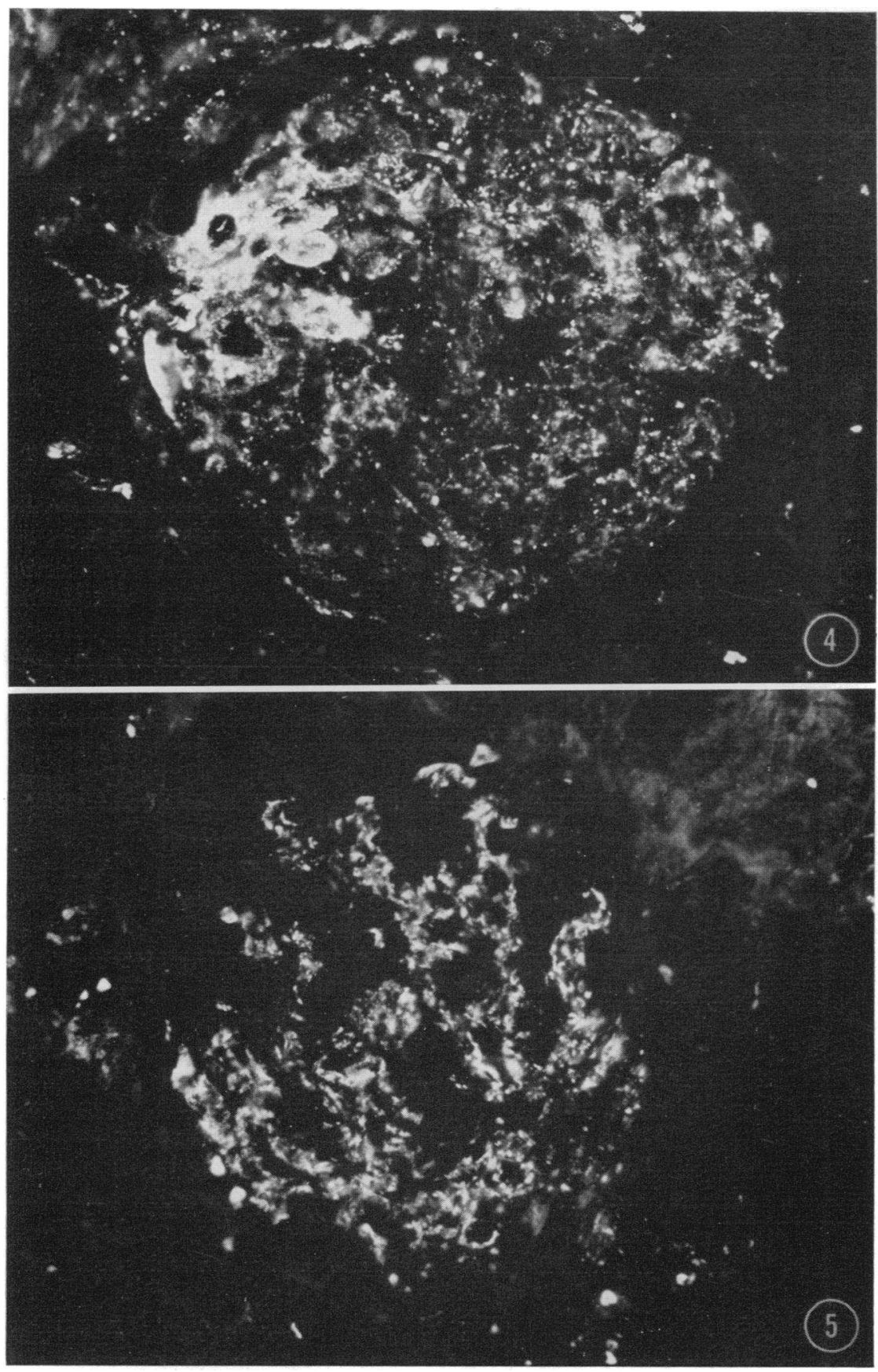

FIGURE 4 Representative glomerulus from a patient with early acute poststreptococcal glomerulonephritis. Anti-properdin antiserum stains "humps" on the basement membrane. Fluorescent microscopy. $\times 750$.

FIGURE 5 Kidney biopsy from a patient with acute poststreptococcal glomerulonephritis obtained $4 \mathrm{wk}$ after onset of hematuria. Characteristic mesangial staining for properdin (shown) which is identical with the staining for $\beta_{1} \mathrm{C}$. Flourescent microscopy. $\times 600$. 
of $\beta_{1} \mathrm{C}$ and a trace positive deposition of properdin in the mesangial stalk and in the glomerular arterioles. Tissues showing "trace" staining are not included in Table II. In no other cases was properdin found. No glomerular deposition of properdin was observed in kidney tissue from patients with vasculitis, periarteritis nodosa, Goodpasture's syndrome, hemolytic anemia-uremia syndrome, and cryoglobulinemia with nephritis.

\section{DISCUSSION}

The isolation of properdin and several components of the classical complement sequence in a chemically and immunologically pure form has now made it possible to attack the problems of inactivation of complement independent of antigen-antibody complexes with more confidence than was possible when properdin was first described. Highly purified human properdin was shown by Pensky et al (4) to be unrelated to the immunoglobulins on the basis of physical, chemical, and antigenic properties and by its functional behavior in immunologic systems. Antigenic determinants in common with C1q, C3, C4, or C5 were not found. The properdin antiserum in the present investigation often did not react in immunofluorescence with renal tissue that stained for IgG, IgM, albumin, fibrin, C1q, C4, and C3 and its staining properties were abolished only by absorptions with properdin and not affected by the addition of $\beta_{1} \mathrm{C}$, $\mathrm{Clq}$, and C5. Not unexpectedly, it was found that the antiserum to $\beta_{1} \mathrm{C}$ prepared after absorption of complement to zymosan (anti- $\beta_{1} C-Z$ ) contained antibodies to both $\beta_{1} \mathrm{C} / \beta_{1} \mathrm{~A}$ and properdin.

This investigation has shown that in all cases of AGN and HCGN that we have studied, properdin is deposited in the glomeruli. In other diseases, even with intense immunofluorescence for $\beta_{1} \mathrm{C}$ and/or IgG, properdin was, as a rule, not found (Table II). The only clear exceptions are 2 out of 19 end stage kidneys, and 1 kidney with SLE nephropathy that also showed rather advanced histological destruction of the glomeruli.

The immunopathology of AGN and HCGN differs from that observed in systemic lupus erythematosus and in experimental immune complex nephritis in three important ways. Firstly, immunoglobulins are often not
TABLE II

Glomerular Localization of Properdin in Patients with Renal Disease

Disease

Chronic membranoproliferative glomerulonephritis with low C3

Chronic membranoproliferative glomerulonephritis (normal serum complement)

Acute poststreptococcal glomerulonephritis

Subacute glomerulonephritis

Membranous glomerulonephritis

Chronic glomerulonephritis (normal serum complement)

Systemic lupus erythematosus

Anaphylactoid purpura

All other kidney diseases with positive immunofluorescence

Nephrosis, idiopathic or congenital

Transplant rejection

End stage kidney disease

Normal kidneys

* Number of patients with significantly positive immunofluorescence for properdin in the glomeruli/total number studied. Trace positive results are not included (see text).

found deposited in the glomeruli in AGN and HCGN $(5,6,18)$. Although this phenomenon can be observed late in "one shot" serum sickness in rabbits (19), it is not seen acutely or in the chronic disease model. Secondly, there is depression of C3 and terminal complement components in the serum with $\mathrm{C} 1, \mathrm{C} 4$, and $\mathrm{C} 2$ not being significantly decreased (20). Thirdly, a heatlabile anticomplementary factor is present in sera from patients with AGN and HCGN that can inactivate guinea pig C3 (21) or in the case of HCGN human C3 (22). In the latter disease a cofactor is lacking which is present in normal human serum or the pseudoglobulin fraction prepared from normal serum. Properdin, being a euglobulin, is probably distinct from this cofactor. The possibility that properdin may be one of the factors involved in this inactivation of $\mathrm{C} 3$ became

TABLE III

Immunopathological Studies in Acute and Chronic Glomerulonephritis with Hypocomplementemia

\begin{tabular}{|c|c|c|c|c|c|c|c|}
\hline & \multicolumn{7}{|c|}{ Glomerular deposits demonstrated by immunofluorescent microscopy } \\
\hline & IgG & IgM & Fibrin & C1q & $\mathrm{C4}$ & $\mathbf{C 3}$ & Properdin \\
\hline $\begin{array}{l}\text { Chronic membranoproliferative glomerulonephritis } \\
\text { with low C3 }\end{array}$ & $4 / 8^{*}$ & $3 / 6$ & $6 / 8$ & $3 / 5$ & $4 / 5$ & $8 / 8$ & $8 / 8$ \\
\hline Acute poststreptococcal glomerulonephritis & $5 / 8$ & $0 / 5$ & $4 / 8$ & $0 / 6$ & $1 / 5$ & $8 / 8$ & $8 / 8$ \\
\hline
\end{tabular}

* Number of patients with deposits/total number of patients studied. 
more likely after the demonstration by Gewurz et al (3) that serum levels of properdin were low in all cases of AGN studied and in about one-half of the cases of HCGN. The in vivo inactivation of $\mathrm{C} 3$ in those two diseases were challenged by Alper, Levin and Rosen (23) who found no evidence for increased turnover of isotope labeled $\beta_{1} \mathrm{C}$ in patients with HCGN. However, later results by Herdman et al (6) obtained with the same technique showed that these patients do have an increased consumption of $\beta_{1} C$ in vivo.

Sera from patients with HCGN and AGN have a diminished ability to react with endotoxic lipopolysaccharide with a reduction of the $\mathrm{C} 3$ activity and decreased formation of a C3-fixing intermediate (24). Properdin may be involved in those reactions. It is interesting to note that in many of our patients, Clq and $\mathrm{C} 4$ seem to be present in the glomeruli. However, the specificity of the antisera to $\mathrm{Clq}$ and $\mathrm{C} 4$, when used for indirect immunofluorescence, could not be tested exhaustively due to lack of reagents and kidney biopsy tissue, and the results obtained must be regarded as preliminary.

At the present time, it is not clear whether properdin is directly involved in the inactivation of $\mathrm{C} 3$ in $\mathrm{AGN}$ and HCGN. Properdin inactivates C3 in vitro after formation of a complex with polysaccharides such as zymosan or inulin (25). It is tempting to speculate that polysaccharides in streptococci or other microorganisms could activate the properdin system in vivo. Another possibility is that the glomerular capillary filter is changed in AGN or HCGN in such a way that its carbohydrate groups are accessible for interaction with properdin. Additional factors may also be involved in the properdin system and could be of importance in these diseases.

The immunological injury to the kidney in glomerulonephritis, recently reviewed by Dixon (26) and by Fish, Michael, and Good (27), is generally regarded as being caused by deposition in the glomeruli of either antibodies to glomerular basement membrane, as in Goodpasture's disease, or of antigen-antibody complexes. On the basis of experimental analysis and correlative morphologic techniques, antigen-antibody complexes have been considered pathogenetically important in certain diseases, namely SLE, AGN, chronic glomerulonephritis, nephritis in chronic infections, and experimental nephritis induced by foreign proteins. However, in HCGN the antigen is unknown, IgG can often not be found in the glomerulus, and the selective depression of the terminal complement components differs from the reduction of all complement components, caused by the reaction of antigen-antibody complexes with serum. The finding of a deposition of properdin together with $\mathrm{C} 3$ in the kidney in HCGN and AGN may point to a mechanism other than that involving antigen- antibody complexes for complement activation and glomerular injury.

Note added in proof. Since the completion of this manuscript, we have noticed in the glomeruli of two patients with SLE, a weak staining for properdin together with a heavy deposition of $\beta_{1}$ C.

\section{ACKNOWLEDGMENTS}

We are grateful to Doctors H. Gewurz and R. Pickering for helpful discussion during this work; and to Dr. $H$. Müller-Eberhard, U. Nilsson, R. Stroud, and C. West who have provided reagents and antisera. We wish to thank Mrs. F. Knudson and Mrs. L. Lang for their expert technical assistance.

This work supported by grants from the National Institutes of Health (AM 12375, HE 05662, HE 06134, H 1263C) ; The American Heart Association; and the Minnesota Heart Association.

Dr. N. Gunnar Westberg was supported by the Minnesota Heart Association, the Insurance Company Forenade Liv, Sweden, and the Hesselman Fund, Sweden.

\section{REFERENCES}

1. Pillemer, L., L. Blum, I. H. Lepow, O. A. Ross, E. W. Todd, and A. C. Wardlaw. 1954. The properdin system and immunity. I. Demonstration and isolation of a new serum protein, properdin, and its role in immune phenomena. Science (Washington). 120: 279.

2. Pillemer, L. 1955. The properdin system. Trans. N. Y. Acad.Sci. 17: 526 .

3. Gewurz, H., R. J. Pickering, G. Naff, R. Snyderman, S. E. Mergenhagen, and R. A. Good. 1969. Decreased properdin activity in acute glomerulonephritis. Int. Arch. Allergy Appl. Immunol. 36: 592.

4. Pensky, J., C. F. Hinz, Jr., E. W. Todd, R. J. Wedgwood, J. T. Boyer, and I. H. Lepow. 1968. Properties of highly purified human properdin. J. Immunol. 100: 142.

5. Michael, A. F., R. C. Herdman, A. J. Fish, R. J. Pickering, and R. L. Vernier. 1969. Chronic membranoproliferative glomerulonephritis with hypocomplementemia. Transplant. Proc. 1 : 925.

6. Herdman, R. C., R. J. Pickering, A. F. Michael, R. L. Vernier, A. J. Fish, H. Gewurz, and R. A. Good. 1970. Chronic glomerulonephritis associated with low serum complement activity (chronic hypocomplementemic glomerulonephritis). Medicine (Baltimore). 49: 207.

7. Michael, A. F., Jr., K. N. Drummond, R. A. Good, and R. L. Vernier. 1966. Acute poststreptococcal glomerulonephritis: Immune deposit disease. J. Clin. Invest. 45: 237.

8. Gajl-Peczalska, K. J., A. J. Fish, H. J. Meuwissen, D. Frommel, and R. A. Good. 1969. Localization of immunological complexes fixing $\beta_{1} \mathrm{C}$ (C3) in germinal centers of lymph nodes. J. Exp. Med. 130: 1367.

9. Wood, B. T., S. H. Thompson, and G. Goldstein. 1965. Fluorescent antibody staining. III. Preparation of fluorescein-isothiocyanate-labeled antibodies. J. Immunol. 95 : 225.

10. Müller-Eberhard, H. J., U. Nilsson, and T. Aronsson. 1960. Isolation and characterization of two $\beta_{1}$-glycoproteins of human serum. J. Exp. Med. 111: 201.

11. Mardiney, M. R., Jr., and H. J. Müller-Eberhard. 1965. Mouse $\beta_{1 \mathrm{c}}$-globulin: Production of antiserum and char- 
acterization in the complement reaction. J. Immunol. 94: 877.

12. Müller-Eberhard, H. J., and C. E. Biro. 1963. Isolation and description of the fourth component of human complement. J. Exp. Med. 118: 447.

13. West, C. D., S. Winter, J. Forristal, J. M. McConville, and N. C. Davis. 1967. Evidence for in vivo breakdown of $\boldsymbol{\beta}_{\mathbf{1 c}}$-globulin in hypocomplementemic glomerulonephritis. J. Clin. Invest. 46: 539.

14. Ouchterlony, Ö. 1958. Diffusion-in-gel methods for immunological analysis. Progr. Allergy. 5: 1.

15. Scheidegger, J. J. 1955. Une micro-méthode de l'immuno-électrophorèse. Int. Arch. Allergy Appl. Immunol. $7: 103$.

16. Hinz, Jr., C. F., and A. M. Mollner. 1963. Studies on immune human hemolysis. III. Role of $11 \mathrm{~S}$ component in initiating the Donath-Landsteiner reaction. $J$. Immunol. $91: 512$.

17. Nilsson, U. R., and H. J. Müller-Eberhard. 1965 Isolation of $\beta 1 \mathrm{~F}$-globulin from human serum and its characterization as the fifth component of complement. J. Exp. Med. 122 : 277.

18. Fish, A. J., R. C. Herdman, A. F. Michael, R. J. Pickering, and R. A. Good. 1970. Epidemic acute glomerulonephritis associated with type 49 streptococcal pyoderma II. Correlative study of light, immunofluorescent and electron microscopic findings, Amer. J. Med. 48: 28.

19. Fish, A. J., A. F. Michael, R. L. Vernier, and R. A. Good. 1966. Acute serum sickness nephritis in the rabbit: An immune deposit disease. Amer. J. Pathol. 49: 997.
20. Gewurz, H., R. J. Pickering, S. E. Mergenhagen, and R. A. Good. 1968. The complement profile in acute glomerulonephritis, systemic lupus erythematosus and hypocomplementemic chloric glomerulonephritis: contrasts and experimental correlations. Int. Arch. Allergy Appl. Immunol. 34 : 556.

21. Pickering, R. J., H. Gewurz, and R. A. Good. 1968. Complement inactivation by serum from patients with acute and hypocomplementemic chronic glomerulonephritis. J. Lab. Clin. Med. 72: 298.

22. Spitzer, R. E., E. H. Vallota, J. Forristal, E. Sudora, A. Stitzel, N. C. Davis, and C. D. West. 1969. Serum C'3 Lytic System in patients with glomeruplonephritis. Science (Washington). 164: 436.

23. Alper, C. A., A. S. Levin, and F. S. Rosen. 1966. Beta1C-globulin: metabolism in glomerulonephritis. Science (Washington). 153: 180.

24. Gewurz, H., R. J. Pickering, J. Fong, N. N. Day, A. F. Michael, R. L. Vernier, and R. A. Good. 1970. Complement consumption in sera of patients with acute and hypocomplementemic glomerulonephritis. Amer. Ped. Soc. Proc. 169. (Abstr.)

25. McNall, E. G. 1957. Assay of properdin in biological fluids. Proc. Soc. Exp. Biol. Med. 96: 399.

26. Dixon, F. J. 1968. The pathogenesis of glomerulonephritis. Amer. J. Med. $44: 493$.

27. Fish, A. J., A. F. Michael, and R. A. Good, 1970. Pathogenesis of glomerulonephritis. In Diseases of the Kidney. M. B. Strauss, and L. G. Welt, editors. Little, Brown and Company, Boston, Mass. 2nd edition. (in press). 